\title{
Conductive Hearing Loss with an Intact Tympanic Membrane: Diagnosed Approaches
}

\section{Sana Mallouk*, Sara Halily, Yasser Hammouda, Youssef Oukessou, Sami Rouadi, Reda Abada, Mohamed Mahtar, Mohamed Roubal}

Department of Otorhinolaryngology, King Hassan II University Hospital, Casablanca, Morocco

\section{Email address:}

sanaa.mallouk@gmail.com (S. Mallouk), halilydoc@gmail.com (S. Halily), y.hammouda@gmail.com (Y. Hammouda), oukessou 22@gmail.com (Y. Oukessou), samirouadi@gmail.com (S. Rouadi), redaalahabada@gmail.com (R. Abada), mahtar.mohamed1@gmail.com (M. Mahtar),med.roubal@gmail.com (M. Roubal)

${ }^{*}$ Corresponding author

\section{To cite this article:}

Sana Mallouk, Sara Halily, Yasser Hammouda, Youssef Oukessou, Sami Rouadi, Reda Abada, Mohamed Mahtar, Mohamed Roubal. Conductive Hearing Loss with an Intact Tympanic Membrane: Diagnosed Approaches. International Journal of Otorhinolaryngology. Vol. 6, No. 2, 2020, pp. 26-30. doi: 10.11648/j.ijo.20200602.12

Received: August 5, 2020; Accepted: August 17, 2020; Published: September 16, 2020

\begin{abstract}
Objective: Our study aims to analyze the epidemiological, clinical, radiological, and etiological aspects of this disease. Methods: We reviewed data from patients who consulted the same seigneur for hearing loss and in whom the otoscopic examination was normal with a conductive hearing loss objectively confirmed on tonal audiometry, for a period of one year (from October 2018 to October 2019). Epidemiological, clinical and paraclinical data were collected and recorded onfarm records. Results: The average age is 36 years. The M/F sex ratio was 1.5 in favor of the men. None of our patients reported a personal otologic history. Three patients reported a notion of head trauma and a similar case in the family was found in four patients. Hearing loss is the most frequent reason for consultation. Otoscopic examination shows a normal tympanic membrane without perforation in all patients. CT scan of the temporal bone was performed in all patients (100\%); it was normal in 4 cases (17\%), which required surgical exploration. However, he showed images of otosclerosis in 15 cases $(65 \%)$, an image of congenital cholesteatoma in a single patient, tympanic glomus in a single patient, an incudo-malleolar dislocation in a single patient $(4.5 \%)$, and an incudo-stapedial dislocation in a single patient (4.5\%). Conclusion: Conductive hearing loss (CHL) is a diagnostic challenge for the physician that requires a schematic approach. The diagnosis is based on a combination of clinical and paraclinical arguments.
\end{abstract}

Keywords: Conductive Hearing Loss, Intact Tympanic Membrane, Otoscopic Examination, CT Scan of Temporal Bone

\section{Introduction}

Conductive hearing loss (CHL) with an intact tympanic membrane is defined as a decrease in hearing due to a defect in the transmission of the acoustic wave between the sound source and the cochlea, with the normal tympanic membrane. The damage can occur: in the external ear but most often in the middle ear. There are several etiologies to be mentioned, however, otosclerosis is the main cause in adults [1]. The diagnosis is based on a combination of clinical and paraclinical arguments. CT scan is the exam of choice in the management of conductive hearing loss with a normal tympanic membrane $[2,3]$. It allows a positive diagnosis and establishes the etiological diagnosis. However, in many other cases, the CT scan is insufficient to identify abnormal radiological findings, which require surgical exploration [4].

Our study aims to analyze the epidemiological, clinical, radiological, and etiological aspects of this disease.

\section{Materials and Methods}

This is a prospective study of patients who consulted the same seigneur for hearing loss and in whom the otoscopic examination was normal with a conductive hearing loss objectively confirmed on tonal audiometry, for a period of one year (from October 2018 to October 2019). Patients with 
an inflammatory condition (otitis media and cholesteatoma) and a history of otologic surgery were excluded from this study.

Epidemiological, clinical and paraclinical data were collected and recorded on-farm records. 23 patients with transmission hearing loss with normal tympanic membrane were enrolled.

\section{Results}

The average age is 36 years with extremes ranging from 16 to 56 with a predominance of the $20-40$ age group. Our series included 14 men and 9 women. The M/F sex ratio was 1.5 in favor of the men.

None of our patients reported a personal otologic history. Three patients reported a notion of head trauma and a similar case in the family was found in four patients. Hearing loss is the most frequent reason for consultation. $63 \%$ of the cases are associated with tinnitus, $4 \%$ with dizziness, and $4 \%$ with cephalea. Hearing loss is progressive (figure 1). Bilateral in $75 \%$ of cases.

Otoscopic examination shows a normal tympanic membrane without perforation in all patients. A white retrotympanic mass bulging behind an intact tympanic membrane was found in only one patient and a pulsating blue retro-tympanic mass also found in only one case (figure 2). Acoumetry showed conductive deafness in the most cases.

Pure-tone audiometry found conductive hearing loss in all cases with an average air-bone gap of $30 \mathrm{~dB}$. The stapedial reflex was absent in 16 cases.

CT scan of the temporal bone was performed in all patients (figure 3 ). It was normal in 4 cases $(17 \%)$, which required surgical exploration. However, he showed images of otosclerosis in 15 cases $(65 \%)$, an image of congenital cholesteatoma in a single patient, tympanic glomus in a single patient, an incudo-malleolar dislocation (figure 4) in a single patient $(4.5 \%)$, and an incudo-stapedial dislocation in a single patient (4.5\%) (Figure 5).

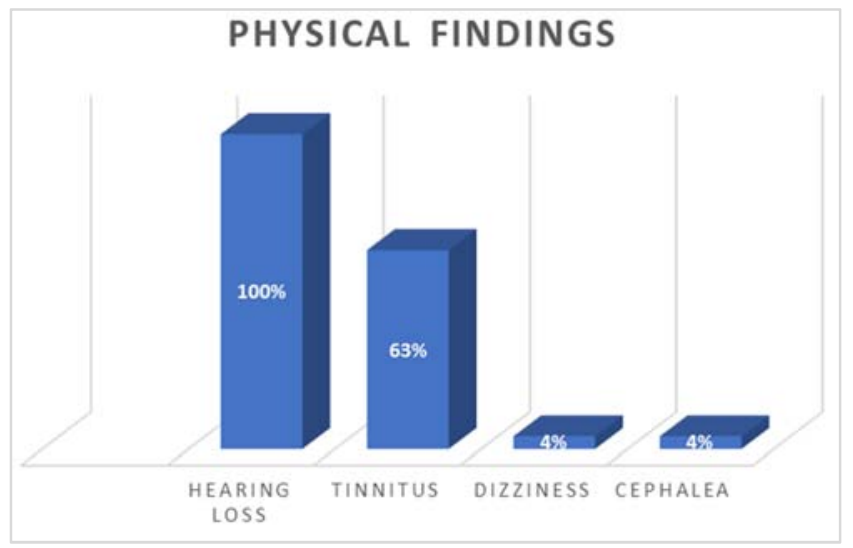

Figure 1. Physical findings.
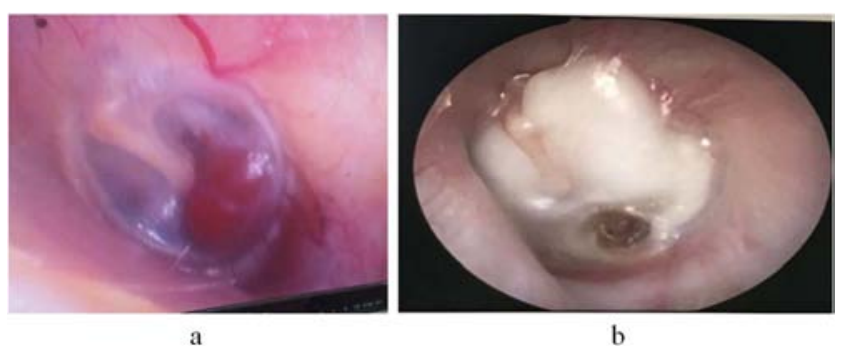

Figure 2. a: otoscopic image of a mass blue behind an intact tympanic membrane evoking Glomus tumor, b: otoscopic image of a mass bulging behind an intact tympanic membrane evoking congenital cholesteatoma.

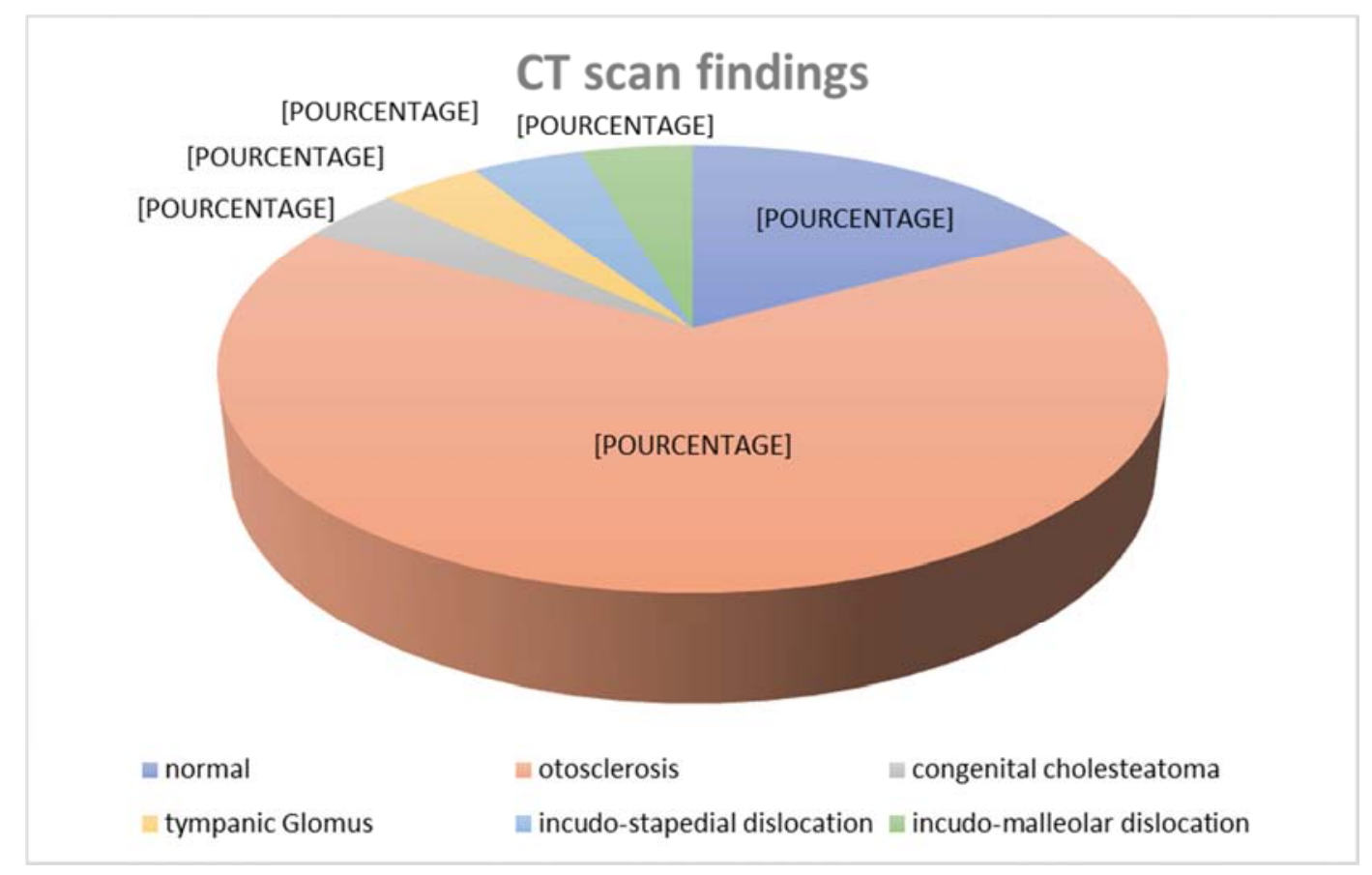

Figure 3. CT scan findings. 


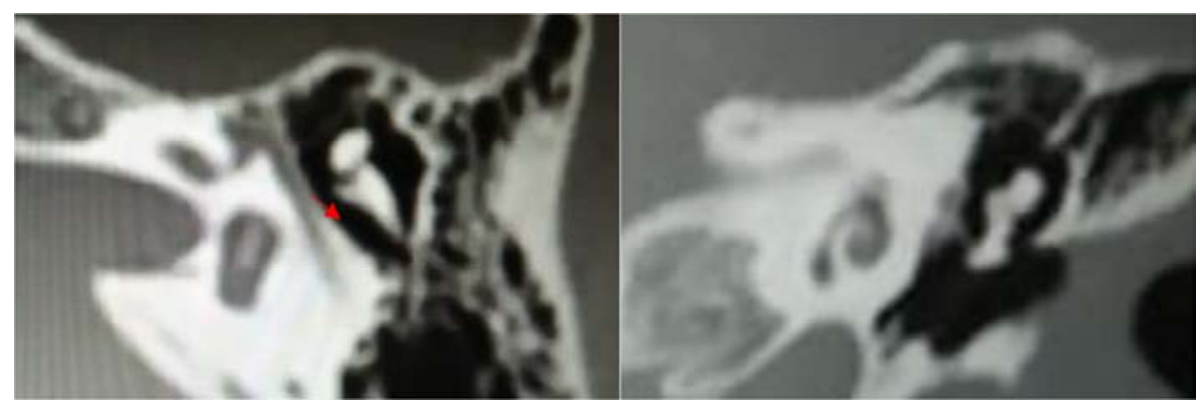

Figure 4. Axial and coronal section of the left temporal bone showing Incudo-malleolar dislocation: Subluxation of the incus relative to the malleus without obvious displacement (arrow).

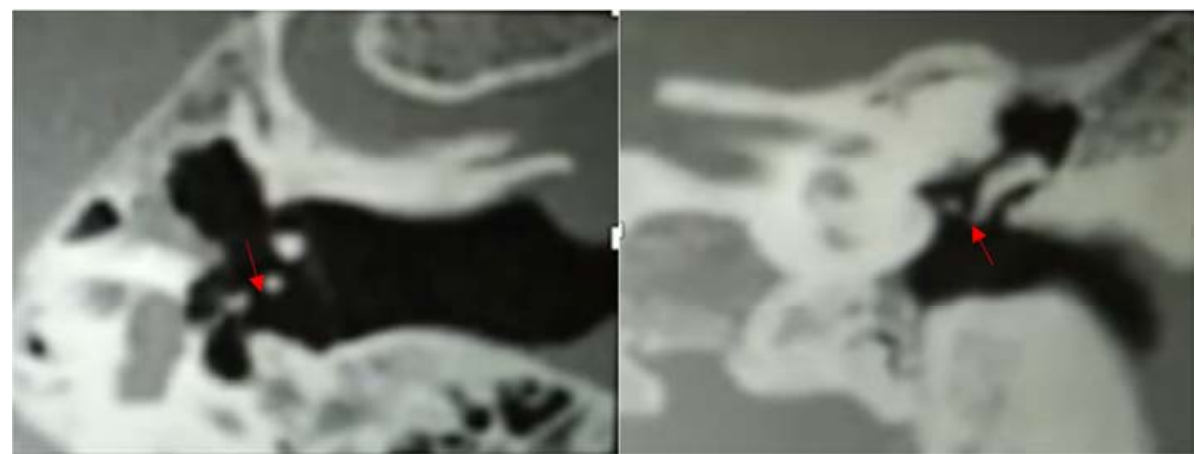

Figure 5. Axial and coronal section of the left temporal bone showing incudostapedial disarticulation: the lenticular process of the incus pulled away from the head of the stapes (arrow).

\section{Discussion}

Conductive hearing loss (CHL) is a frequent reason for ENT consultation, however, it represents a diagnostic challenge especially in patients presenting for (CHL) with intact tympanic membrane as there is no absolute diagnostic test for these patients [4]. Nevertheless, no exact statistics for conductive hearing loss with a normal tympanic membrane. In this type of deafness, the inner ear functions normally. Hearing loss is the result of a perturbation of the propagation of sound waves from the outer environment to the sensory cells of the inner ear. It is often curable by medical or surgical means. However, in children, it is a diagnostic and therapeutic emergency because of its impact on language development.

Clinically, it is important to search for the causes of conductive hearing loss because some cases of deafness require surgery. But, in other cases, a conservative approach is recommended [4]. A positive diagnosis is based on a good medical interrogation, permitting to specify the chronology and the evolution of the symptoms, as well as to determine the social and relational gene caused. It is also necessary to look for similar cases in the family, which can direct the diagnosis towards otosclerosis, an antecedent of head trauma that can help the diagnosis towards a trauma of the ossicular chain. In our series, a similar case in the family was found in four patients and three patients reported cranial trauma. The age of development of conductive hearing loss with an intact tympanic membrane is difficult to determine. The most studies, the diagnosis has been made mainly in the second decade of life $[4,5]$. In our study, the average age is 36 years with extremes from 16 to 56 years with a predominance of the 20-40-year age range.

A well-performed otoscopic examination, studying the external ear canal, the tympanic membrane and the middle ear, can guide the diagnosis. Usually, the tympanic membrane is normal in appearance, in some situations, a whitish mass behind an intact tympanic membrane is suggestive of a congenital cholesteatoma and a pulsating bluish mass is suggestive of a tympanic glomus. Occasionally, a retro tympanic pink spot can be found, corresponding to the Schwartz sign, which is a hyper vascularization of the promontory, which is specific to otosclerosis [6]. In our study, all patients had a normal tympanic membrane without perforation $(100 \%)$. However, a whitish retro tympanic mass was found in only one patient and a pulsatile bluish retro tympanic mass was also found in only one patient.

The tuning fork test objective a Weber test lateralized on the deaf side and a negative Rinne test, which affirms the transmissibility of deafness [7].

A pure-tone audiogram is an essential assessment test, both for positive diagnosis and for evaluation of severity and monitoring of evolution, CHL is usually mild to moderate and may be unilateral or bilateral. But it provides little detail about the pathology or the cause of conductive deafness [7]. A conventional tympanogram is generally normal with an absent stapedial reflex, but it does not distinguish between mastoid and ossicular chain abnormalities. Recently, Wideband Tympanometry (WBT) has been more and more used to surmount the limitations of conventional tympanogram [8]. WBT uses absorbance, in contrast to the conventional tympanogram which uses admittance. It can 
help to differentiate between patients with a tympanic perforation alone and patients with an associated ossicular chain problem. It can also provide more information about patients with mastoid problems [8,9]. Data from a recent study justifies the larger applications of WBT, as it can differentiate between different conductive hearing loss conditions [10]. Otoacoustic emissions have low diagnostic value, the presence of conductive hearing loss is sufficient to reduce the clinical utility of distortion product otoacoustic (DPOAE). But, through a strategic modification of primary tone levels, the clinician can improve the measurement of DPOAE [11].

$\mathrm{CT}$ is the first imaging modality used to evaluate a patient with conductive hearing loss with an intact tympanic membrane [2, 3]. In the presence of CHL with a normal tympanic membrane, otosclerosis should be suspected, but other pathologies should not be forgotten. Thus, the round window, the integrity of the ossicles, and the upper semicircular canal should be well examined. High-resolution CT also permits a study of the extent of the disease, anatomical variants, and associated complications, for better therapeutic management [2]. Nevertheless, to establish an accurate diagnosis, CT imaging results should always be correlated with clinical and otoscopic findings. Certainly, the CT scan is the examination of choice in the management of conductive hearing loss with a normal tympanic membrane, however, in many other cases, it is insufficient to identify abnormal radiological findings, which require surgical exploration [4].

In our study, the CT scan was normal in $17 \%$ of the cases which required surgical exploration for these patients.

Several etiologies are to be evoked however otosclerosis represents the main cause in adults [1]. Otosclerosis is a primary osteodystrophy of the endochondral bone of the otic capsule (temporal bone), it is a genetic disease with autosomal dominant transmission. [7]. In general, the diagnosis is simple and is based on the presence of a normal otoscopic examination, a typical audiogram (Conductive hearing loss on all frequencies with air-bone gap of 60-65 dB and Carhart notch at $2000 \mathrm{~Hz}$ ) and the absence of stapedial reflexes $[12,13]$. Currently, high-resolution computed tomography (HRCT) confirms the diagnosis by visualizing otosclerotic foci as hypodense or radiolucent foci and classifying them into four stages according to the Veillon classification, it also allows an evaluation of the prognosis and elimination of other pathologies, as additionally, a presurgical anatomical assessment can be carried out [14]. However, the absence of foci does not exclude the possibility of stapes fixation by sclerotic focus [4].

In our study, the CT scan established the diagnosis of otosclerosis in $65 \%$ of cases.

The second common cause of $\mathrm{CHL}$ is ossicular chain trauma, it accounts for about $1.4 \%$ of all head trauma and $37 \%$ of all fractures of the petrous pyramid [15]. According to Brodie and Thompson's study, 21\% of patients with documented hearing loss after a temporal bone fracture had conductive hearing loss [16]. Longitudinal fractures of the temporal bone are usually observed with fractures of the ossicular chain [19]. Generally, CHL is related to the tympanic membrane or hemotympanum rupture, but if deafness persists for longer than about one month, ossicular damage should be suspected [17]. Dislocation is the most typical type of injury and occurs more often than fracture, five types of dislocations exist: incudostapedial joint separation, incus dislocation, incudomalleal joint separation, stapediovestibular dislocation and incudomalleal complex dislocation [18]. The most common lesion of the ossicular chain is incudostapedial joint separation [19]. However, it cannot be visible on the scanner, but usually, a gap between the lenticular process of the incus and the head of the stapes is objective.

In our study, the CT scan showed an incudo-stapedial dislocation in a young patient with a history of head trauma following a public road accident, in whom otoscopic examination showed a hemotympanic and pure-tone audiometry found conductive hearing loss with a loss of $70 \mathrm{db}$. Also, the CT scan showed an i incudo-malleolar dislocation in a young patient with no history of head trauma.

All tumors of the middle ear can cause conductive hearing loss by blockage of the ossicular chain. Primary cholesteatoma and tympanic paraganglioma are the two most common causes. Usually, a good otoscopic examination will suggest the diagnosis. Generally, the clinical presentation is a middle-aged woman presenting with CHL associated with pulsating tinnitus [20]. CT scan shows a focal flat-based mass on the cochlear promontory of the middle ear without bone erosion [2]. However, Congenital cholesteatoma (CC) is one of the most common causes of conductive deafness especially in the pediatric population which is the main reason for consultation [21]. Usually the diagnosis of CC is made by clinical and otoscopic examination, it presents as a white mass behind a normal tympanic membrane, without a previous history of perforation, ear discharge, or ear surgery [22]. A CT scan of the temporal bone is performed to evaluate extension, to explore complications, and to identify anatomical variants in preparation for surgery.

LCH with intact MT could be observed in superior semicircular canal dehiscence syndrome (SCDS). This syndrome is characterized by the formation of a "third window" between the superior semicircular canal and the middle cranial fossa, caused by a bony defect of the canal [23]. This results in a loss of acoustic energy responsible for conductive or mixed deafness [24]. CT is the radiological examination of choice; it allows us to determine with precision the dehiscent segment of the semi-circular canals [2]. In our series, only one patient had an image of dehiscence of the superior semicircular canal on the CT scan of the temporal bone.

Finally, minor aplasia is the first diagnosis to evoke in a pediatric patient who presents for congenital conductive hearing loss.

It is defined as a malformation of the middle ear isolated or associated with minor abnormalities of the external ear (pinna and/or external ear canal) or other general malformations. Good classification of middle ear 
malformations is important to determine the surgical approach and to determine the prognosis [25]. In our study, no cases of middle ear deformities were noted.

\section{Conclusion}

Deafness is a sensorial handicap which leads to depression, social isolation and reduced cognitive functions, especially in children. This is why screening and early treatment of deafness is essential. Conductive hearing loss (CHL) is a diagnostic challenge for the physician that requires a schematic approach. Otosclerosis is the most common cause.

Imaging assessment of CHL is guided primarily by clinical and otoscopic findings.

High-resolution computed tomography (CT) plays an essential role in the evaluation of CHL. Sometimes, the CT scan is insufficient to identify abnormal radiological findings, which require surgical exploration.

\section{References}

[1] Espitalier F, Durand N, Boyer J, Gayet-Delacroix M, Malard O, Bordure P. Stratégie diagnostique devant une surdité de l'adulte. EMC (Elsevier Masson SAS), Oto-rhino laryngologie. 2012; 7 (2): 1-12.

[2] Subramanian, M., Chawla, A., Chokkappan, K., Lim, T., Shenoy, J. N., \& Chin Guan Peh, W. (2018). High-Resolution Computed Tomography Imaging in Conductive Hearing Loss: What to Look for? Current Problems in Diagnostic Radiology, 47 (2), 119-124.

[3] Curtin, H. D. (2016). Imaging of Conductive Hearing Loss With a Normal Tympanic Membrane. American Journal of Roentgenology, 206 (1), 49-56.

[4] Kim SH, Cho YS, Kim HJ, Kim HJ. Operative findings of conductive hearing loss with intact tympanic membrane and normal temporal bone computed tomography. Eur Arch Otorhinolaryngol 2014; 271: 1409-14.

[5] Thomeer HG, Kunst HP, Cremers CW. Congenital stapes ankylosis associated with another ossicular chain anomaly: surgical results in 30 ears. Arch Otolaryngol Head Neck Surg 2011; 137: 935-41.

[6] Lippy WH, Berenholz LP. Pearls on otosclerosis and stapedectomy. Ear Nose Throat J. 2008; 326-328.

[7] Thomassin JM, Collin M, Bailhache A, Dessi P, Rodriguez F, Varoquaux A. Otospongiose. EMC (Elsevier Masson SAS), Oto-rhino-laryngologie. 2010: 1-15. 20-195-A-10.

[8] Terzi S, Ozgur A, Erdivanli OC, Coskun ZO, Ogurlu M, Demirci M, et al. Diagnostic value of the wideband acoustic absorbance test in middle-ear effusion. J Laryngol Otol 2015; 129: $1078-84$.

[9] Feeney MP, Grant IL, Marryott LP. Wideband energy reflectance measurements in adults with middle-ear disorders. J Speech Lang Hear Res 2003; 46: 901-11.
[10] Kim, S. Y., Han, J. J., Oh, S. H., Lee, J. H., Suh, M., Kim, M. H., \& Park, M. K. (2018). Differentiating among conductive hearing loss conditions with wideband tympanometry. Auris Nasus Larynx.

[11] Kreitmayer, C., Marcrum, S. C., Picou, E. M., Steffens, T., \& Kummer, P. (2019). Subclinical Conductive Hearing Loss Significantly Reduces Otoacoustic Emission Amplitude: Implications for Test Performance. International Journal of Pediatric Otorhinolaryngology.

[12] Virk JS, Singh A, Lingam RK. The role of imaging in the diagnosis and management of otosclerosis. Otol Neurotol. 2013; 34 (7): e55-e60.

[13] Goodhill V, Haris I, Canalis R. Otosclerosis. In: Canalis R, Lambert PR, eds. The Ear: Comprehensive Otology. Philadelphia, PA: Lippincott Williams and Wilkens; 2000: 467-487.

[14] Wolfovitz, A., \& Luntz, M. (2018). Impact of Imaging in Management of Otosclerosis. Otolaryngologic Clinics of North America, 51 (2), 343-355.

[15] Bonafé A, Laval C, Arru Ph, Manelfe C, Temporal bone fractures. Rivista di Neuroradiologia, 1995; 8: 847-54.

[16] Brodie HA, Thompson TC. Management of complications from 820 temporal bone fractures. Am J Otol 1997; 18: 18897.

[17] Juliano AF, Ginat DT, Moonis G. Imaging review of the temporal bone: Part II. Traumatic, postoperative, and noninflammatory nonneoplastic conditions. Radiology 2015; 276: $655-72$.

[18] Stephan LA, Isaacson JE. Incudomallear joint separation. Am J Otol 2000; 21: 284-5.

[19] Yetiser, S., Hıdır, Y., Birkent, H., Satar, B., \& Durmaz, A. (2008). Traumatic ossicular dislocations: etiology and management. American Journal of Otolaryngology, 29 (1), 31-36.

[20] Carlson ML, Sweeney AD, Pelosi S, et al. Surgical management of glomus tympanicum: a review of 115 cases over four decades. Otolaryngol Head Neck Surg 2015; 152: $136-42$.

[21] Kojima H, Tanaka Y, Shiwa M. et al. Congenital cholesteatoma clinical features and surgical results. Am J Otolaryngol. 2006; 27 (5): 299-305.

[22] Bennett M, Warren F, Jackson G, Kaylie D (2006) Congenital cholesteatoma: theories, facts, and 53 patients. Otolaryngol Clin N Am 39: 1081-1094.

[23] Minor LB. Clinical manifestations of superior semicircular canal dehiscence. Laryngoscope 2005; 115 (10): 1717-1727.

[24] Li PM, Bergeron C, Monfared A, Agrawal S, Blevins NH. Superior semicircular canal dehiscence diagnosed after failed stapedotomy for conductive hearing loss. Am J Otolaryngol 2011; 32: 441-4.

[25] Yang, F., \& Liu, Y. (2018). Reporting and Description for Congenital Middle Ear Malformations to Facilitate Surgical Management. Annals of Otology, Rhinology \& Laryngology, 000348941879293. 